\title{
Evaluation by quantitative image analysis of anticancer drug activity on multicellular spheroids grown in 3D matrices
}

\author{
AURÉLIE GOMES ${ }^{1}$, ADRIEN RUSSO ${ }^{1}$, GUILLAUME VIDAL ${ }^{2}$, ELISE DEMANGE ${ }^{2}$, PAULINE PANNETIER ${ }^{2}$, \\ ZIED SOUGUIR $^{2}$, JEAN-MICHEL LAGARDE $^{3}$, BERNARD DUCOMMUN $^{1,4}$ and VALÉRIE LOBJOIS ${ }^{1}$ \\ ${ }^{1}$ Institut des Technologies Avancées en sciences du Vivant, Centre National de la Recherche Scientifique, \\ Université Paul Sabatier, Université de Toulouse, 31106 Toulouse; ${ }^{2}$ CELENYS, 76000 Rouen; ${ }^{3}$ IMACTIV-3D, \\ Centre Pierre Potier, 31106 Toulouse; ${ }^{4}$ Centre Hospitalier Universitaire de Toulouse, 31059 Toulouse, France
}

Received May 18, 2016; Accepted August 19, 2016

DOI: $10.3892 / 01.2016 .5221$

\begin{abstract}
Pharmacological evaluation of anticancer drugs using 3D in vitro models provides invaluable information for predicting in vivo activity. Artificial matrices are currently available that scale up and increase the power of such 3D models. The aim of the present study was to propose an efficient and robust imaging and analysis pipeline to assess with quantitative parameters the efficacy of a particular cytotoxic drug. HCT116 colorectal adenocarcinoma tumor cell multispheres were grown in a 3D physiological hyaluronic acid matrix. 3D microscopy was performed with structured illumination, whereas image processing and feature extraction were performed with custom analysis tools. This procedure makes it possible to automatically detect spheres in a large volume of matrix in 96-well plates. It was used to evaluate drug efficacy in HCT116 spheres treated with different concentrations of topotecan, a DNA topoisomerase inhibitor. Following automatic detection and quantification, changes in cluster size distribution with a topotecan concentration-dependent increase of small clusters according to drug cytotoxicity were observed. Quantitative image analysis is thus an effective means to evaluate and quantify the cytotoxic and cytostatic activities of anticancer drugs on 3D multicellular models grown in a physiological matrix.
\end{abstract}

\section{Introduction}

Despite a large attrition rate primarily due to their lack of predictivity for in vivo activity, 2D cellular assays of

Correspondence to: Dr Valérie Lobjois or Professor Bernard Ducommun, Université de Toulouse, Centre Pierre Potier, ITAV-USR3505, 1 Place Pierre Potier, 31106 Toulouse, Cedex 1, France

E-mail: valerie.lobjois@itav.fr

E-mail: bernard.ducommun@itav.fr

Key words: preclinical drug evaluation, 3D models, 3D matrix, microscopy, automatic image quantification pharmacological efficacy, including cytotoxicity assessment, have been extensively used to evaluate novel candidate anticancer drugs at the preclinical stage (1). However, the development and use of 3D models that reproduce the spatial organization of normal or tumor tissue as closely as possible is necessary (2).

Tumor spheroids are of great interest in overcoming this limitation as they recapitulate numerous key features of tissue, thus providing accurate predictions of drug activity in vivo $(3,4)$. Spheroids are either grown in suspension, or in natural or synthetic matrices, and each method has its own respective advantages and disadvantages (5). Liquid-based growth allows very precise control of spheroid size, dynamic control of culture media and is adapted to low-throughput imaging and analytical methods. However, this method of growth does not allow control of the physical and mechanical properties of the environment. By contrast, scaffold-based culture methods allow for easy handling and offer precise environmental control of growth conditions and compatibility with high-throughput screening. However, one technical limitation of the latter method is the heterogeneity of the size of spheres grown in the matrix. This factor results in imaging being limited when using classical microscopy and difficulties in quantifying the effect.

Due to innovations in hydrogel chemistry, a number of options are currently available that provide researchers with synthetic and natural matrices partially mimicking in vivo extracellular matrix (6). These may be used in tissue engineering as well as to evaluate the growth of 3D cell aggregates $(7,8)$. Drug activity evaluation in medium-scale assays using multiwell plates containing such matrices often relies on a global assessment of the effects by a cell viability readout. Consequently, these approaches do not allow researchers to consider and explore the 3D aspect of the response to drug treatment, thereby restricting the parameters that may be analyzed and the possibility of investigating the mechanisms of action of a given candidate drug. A major limitation of fast imaging with wide-field fluorescence microscopy is the poor quality of the images obtained. However, the use of 3D imaging strategies, including confocal and two-photon microscopy, results in a substantial increase in acquisition time, as well as laser illumination-induced bleaching. The poor quality of images 
obtained by conventional wide-field fluorescence microscopy is due to out-of-focus light; spheres located throughout the matrix are illuminated, therefore those above or below the imaging plane appear highly blurred. Structured illumination using ApoTome technology fitted on a conventional wide-field fluorescence microscope enables researchers to obtain images with similar resolution to confocal microscopy (9). The principle is based on projecting a grid onto the in-focus plane of the sample and acquiring at least three raw images with the grid in three different positions in order to identify in-focus areas (10). Combining the three raw images results in a very clear single image, with no out-of-focus fluorescence signal.

Thus, to fully exploit the potential of an experimental approach based on evaluating drug activity on tumor spheres grown in 3D matrices, it is necessary to develop robust and calibrated procedures allowing for perfectly controlled microscopic imaging conditions and accurate quantitative assessment of growth parameters. The present study aimed to develop the necessary 3D imaging and quantitative characterization methods capable of measuring the features of individual tumor spheres in a large volume of matrix.

\section{Materials and methods}

Cell culture and pharmacological treatments. Human HCT116 colorectalcancercells(ATCC; Teddington,UK) were cultured in Dulbecco's modified Eagle's medium $\left(\right.$ DMEM) + Glutamax ${ }^{\mathrm{TM}}$ (Thermo Fisher Scientific, Inc., Waltham, USA) containing $10 \%$ fetal calf serum (Thermo Fisher Scientific, Inc.) with penicillin/streptomycin (Sigma-Aldrich, St. Louis, MO, USA) in a humidified atmosphere of $5 \% \mathrm{CO}_{2}$ at $37^{\circ} \mathrm{C}$. A suspension of cells was inoculated in Biomimesys ${ }^{\circledR}$ (Celenys, Rouen, France), commercially available 96-well plates containing a hyaluronic acid matrix. A total of 50,000 cells were seeded in $25 \mu \mathrm{l}$ of culture media per well. Following incubation at $37^{\circ} \mathrm{C}$, in $5 \% \mathrm{CO}_{2}$ for $30 \mathrm{~min}, 175 \mu \mathrm{l}$ of culture media was added to each well. After 3 days, half of the volume of the culture medium was renewed. Six days following cell seeding, $100 \mu \mathrm{l}$ of culture media per well was replaced by $100 \mu \mathrm{l}$ of culture media containing a $2 \mathrm{X}$ topotecan concentration (Sigma-Aldrich, St. Louis, MO, USA). On each plate, 6 different concentrations were applied with 6 wells treated per concentration. Plates were fixed $72 \mathrm{~h}$ following treatment.

4',6-diamidino-2-phenylindole (DAPI) staining. Hydrogel matrices were fixed for $15 \mathrm{~min}$ at room temperature with $4 \%$ paraformaldehyde in phosphate-buffered saline (PBS). Following 3 washes in PBS, the samples were permeabilized for $6 \mathrm{~min}$ in $0.5 \%$ Triton $\mathrm{X}-100$ solution, washed 3 times in PBS and subsequently incubated with $1 \mu \mathrm{g} / \mathrm{ml}$ DAPI fluorescent nuclear stain for $10 \mathrm{~min}$ prior to PBS wash.

Image acquisition. Image acquisition was performed using an inverted widefield fluorescence microscope, Axio Observer. Z1, with ApoTome fitted with an axiocam 506 mono camera (Zeiss GmbH, Jena, Germany; objective magnification, x10 and numerical aperture 0.3). For each well, 9 tiles with $10 \%$ surface overlap were acquired. For each tile, a 400-500 $\mu \mathrm{m}-\mathrm{z}$ stack with a $20 \mu \mathrm{m}-\mathrm{z}$ step was performed with 3 images corresponding to different ApoTome grid positions per $\mathrm{z}$ position.
A total of $9 \mathrm{z}$-stacks were acquired per well. For each z-stack, ApoTome deconvolution was performed using ZEN software ver. 2012 (Zeiss GmbH).

Image processing pipeline. For each z-stack, a maximum projection was performed and the 9 images obtained per well were stitched together. Image filtering, projections and the stitching process were executed by an ImageJ macro running on a Dell ${ }^{\mathrm{TM}}$ T7X series Workstation equipped with $192 \mathrm{~GB}$ RAM, a Xeon-phi 32 core processor and a 4 GB VRAM NVidia Graphics card. This level of power is required to process large batches of images (>60 megapixels per image). The stitched images were subsequently processed using a MATLAB ${ }^{\circledR}$ script to remove noise and background, set saturation level, and perform Otsu's automatic threshold and morphological dilatation on stitched images (11). Combining the resulting binary mask with the original image made it possible to select only those pixels corresponding to clusters along with those in their close vicinity. This pre-segmented image was then resampled on 10 levels of grey, with the elimination of the first one leading to final segmentation. For the final step of the analysis process, i.e. quantification, MATLAB's built-in methods of clustering and quantification were used exclusively to compute the per cluster projected area and average intensity response.

\section{Results and Discussion}

Matrix-embedded tumor multisphere imaging in a large volume using structured illumination. Fig. 1 summarizes the workflow and the specific procedures reported, starting from the seeding step of cancer cells in the matrix and 3D sphere formation to the final results of the quantification analysis. As illustrated, 96-well plates containing a hydrogel made of cross-linked hyaluronic acid were seeded with HCT116 colon adenocarcinoma cells. It has been demonstrated that cancer cells proliferate actively in such a matrix (12), recapitulating optimal microenvironmental conditions. After 6 days of growth, a number of 3D cell clusters of various shapes and sizes were formed and detected under bright field microscopy (Fig. 1).

To individually characterize a large number of spheres per well, spheres were fixed inside the matrix and stained with DAPI to be detected by fluorescence microscopy. The aim of the present study was to acquire images of a large number of spheres from each of the 96 wells, therefore, multi-position 3D fluorescence microscopy was utilised. Structured illumination was used with an ApoTome fitted to a conventional wide-field fluorescence microscope. This technology is not based on pixel-by-pixel scanning, therefore it allows for a much more rapid z-stack acquisition of all in-depth information from each of the 96 wells. Individual acquisitions consist of a 400-500 $\mu \mathrm{m}$ z-stack, with each $\mathrm{z}$ separated by $20 \mu \mathrm{m}$ (Fig. 1). A movie (available for download at https://mycore. core-cloud.net $/$ public.php?service $=$ files $\& \mathrm{t}=\mathrm{bcd} 82 \mathrm{c} 01 \mathrm{~cd} 45$ e93c3c55ce 5e175cd7cb) presents a z-stack acquisition of a field of view prior to and following ApoTome deconvolution. These data indicate that following ApoTome deconvolution, nuclei of individual spheres are visible and it is possible to identify each sphere. These findings are confirmed with the maximal projection of the z-stack (data not shown), which 


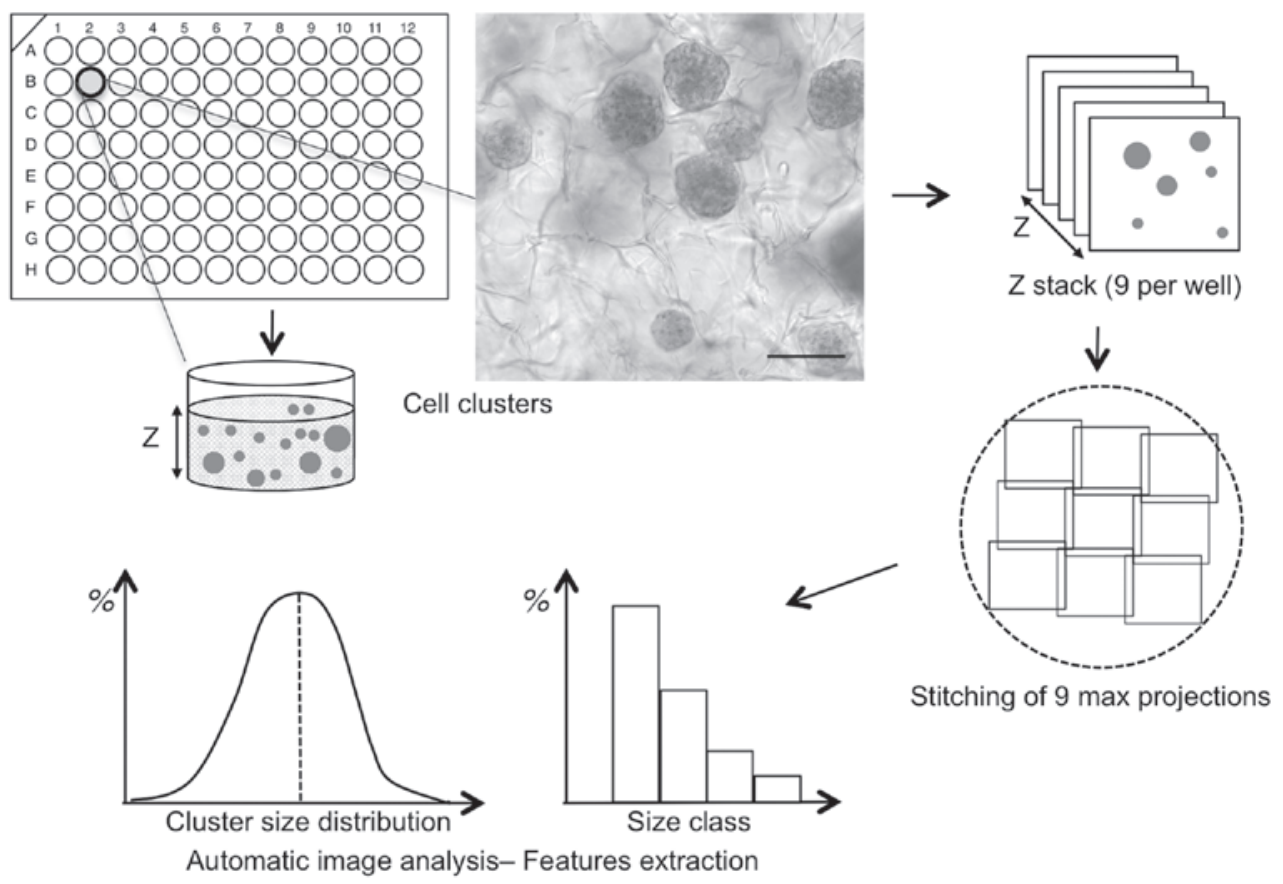

Figure 1. The quantitative image analysis procedure. Cells were cultured in Biomimesys ${ }^{\circledR} 3 \mathrm{D}$ matrix in a 96 -well format. In each well, cells grew as clusters of various sizes. The bright field microscopy image presents the diversity of the size of clusters detected in the matrix 6 days after seeding HCT116 cells (Scale bar, $100 \mu \mathrm{m}$ ). Imaging with a wide-field ApoTome microscope allowed z-stack collection from 9 overlapping fields of view. Stitching together their maximal projections reconstructed the majority of each well. A segmentation, quantification and classification procedure was used to determine cluster size distribution in various experimental conditions, thus allowing drug evaluation with $\mathrm{IC}_{50}$ determination according to morphometric parameters.

makes it possible to qualitatively assess proliferation and count the number of individual cell clusters.

A total of 9 z-stack acquisitions were performed for each well to avoid restricting the analysis to a single field of view and to provide global quantitative information (Figs. 1 and 2A). The nine $400 \mu \mathrm{m} \mathrm{z}$-stack acquisitions with ApoTome covered half of the well area and made it possible to identify each sphere in the volume. To generate the global image of each micro-well (Fig. 2A), the z-stacks of the 9 overlapping tiles of each well were stitched together using a previously described procedure (13). This procedure was applied to wells treated with increasing doses of the DNA topoisomerase inhibitor, topotecan. A total of 6 concentrations were used and 6 wells were treated for each concentration. The stitched images obtained for each well are presented in the mosaic image (Fig. 2B), which represents a virtual multiwell plate reconstructing each well using the aforementioned procedure and including fluorescence information for spheres throughout the matrix in the well's central area $\left(\sim 13 \mathrm{~mm}^{2}\right)$. Cluster size decreases upon treatment with higher concentrations of topotecan, thereby making it possible to qualitatively evaluate the effect of this drug on matrix-embedded multispheres. This representation provides a global view of the experiment, offering an assessment of the effect of a given drug.

Image processing and features extraction. Stitched images from ApoTome acquisition provided all of the cancer cell cluster information available in each well. However, its analysis and interpretation require further processing (Figs. 1 and 3). To this end, a methodology was developed based on image analysis tools and was subsequently applied to images of the mosaic (Fig. 2B). This provided morphometric and multiparametric quantitative data, including cluster size distribution.

The objective was to avoid any manual threshold for image segmentation and to use a single tool to identify large clusters, as well as individual cells. The first step was to filter stitched images and perform a pre-segmentation pass to eliminate background and focus on the upper class of Otsu's segmentation method (11). Extracted areas were then expanded, creating a very coarse mask (binary image, Fig. 3) to identify areas of interest containing cell clusters. Following the combination of this binary mask with the original stitched image, the pixels corresponding to the background were set to 0 , leaving only pixels corresponding to clusters and their near vicinity. The goal here was to avoid removing any of the few multi-sized clusters. To account for the variability of nuclei fluorescence intensities that disrupt traditional segmentation algorithms and to more accurately distinguish clusters from background noise, an intensity scale was used, resampling in 10 levels. Finally, an additional segmentation and refinement was performed from the 9 stitched image tiles to obtain the boundary of each cluster (pink lines on the final image, Fig. 3) and to extract morphometric features.

Procedure application to analyze the cytotoxic effect of topotecan on matrix-embedded multispheres. The quantitative analysis methodology was applied to the stitched images of the wells following topotecan treatment at different concentrations. Fig. 4A illustrates the result of the final segmentation performed on cancer tumor spheroids grown in matrix in a 96-well format and treated with increasing concentrations of the topoisomerase inhibitor topotecan. The left panel presents an untreated control well, where a large number of 3D clusters 
A

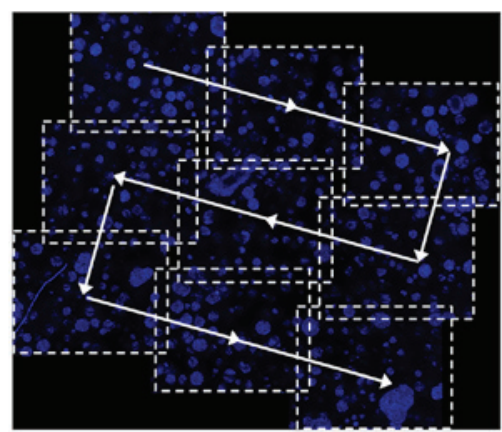

B

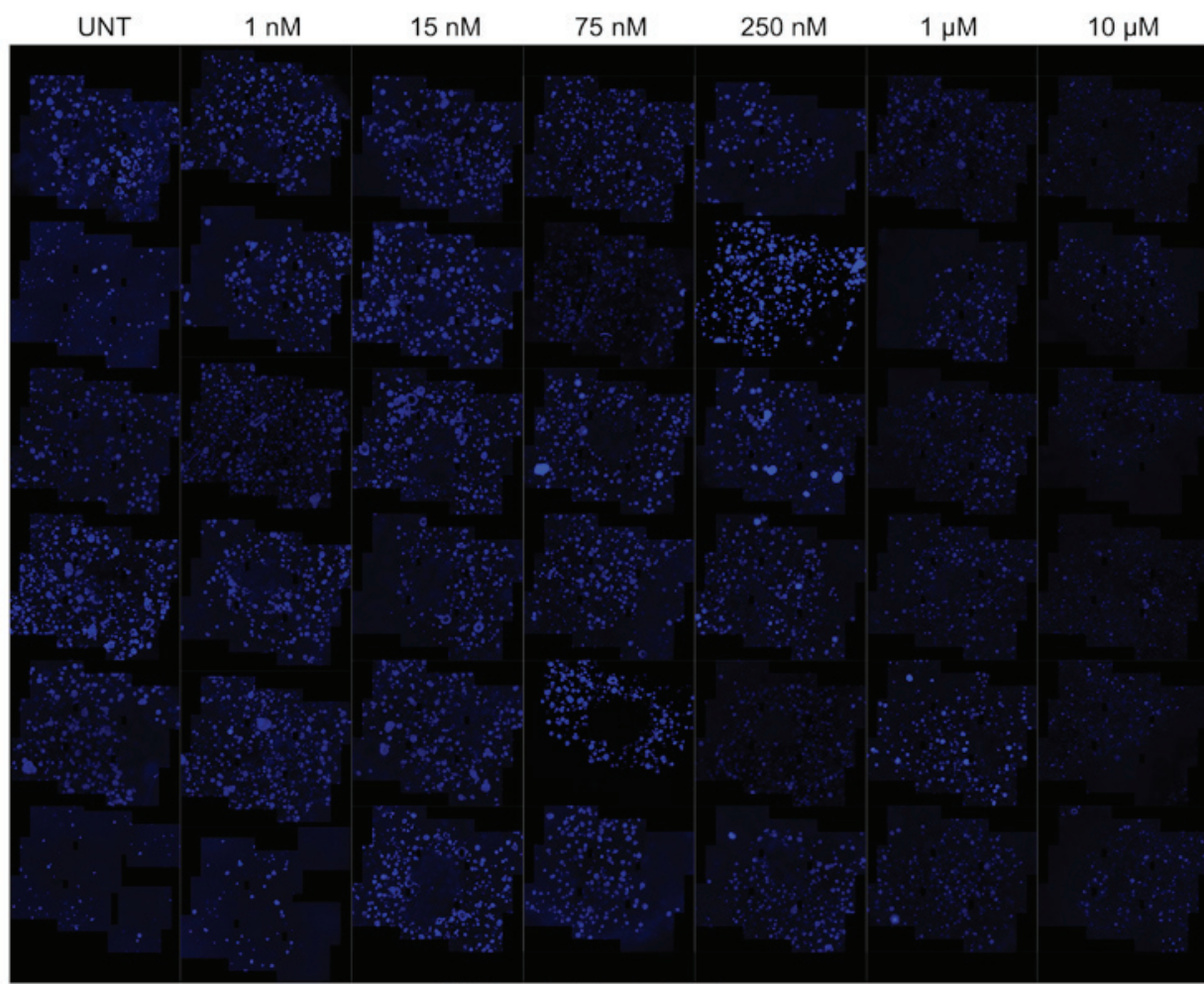

Matching Tiles overlap $\longrightarrow$

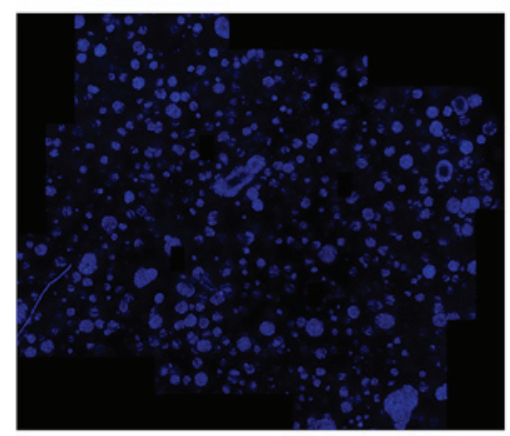

9 fields of view per well

$\mathrm{nM} \quad 1 \mu \mathrm{M}$

Multi-well plate global view

Figure 2. Image z-stack stitching to reconstruct complete well information. A total of 9 image tiles per well were acquired by fluorescence microscopy. (A) Stitching was performed using matching overlaps to reconstruct a global z-stack image of each microwell. (B) Global view of the microwell plate with stitched images for each position.

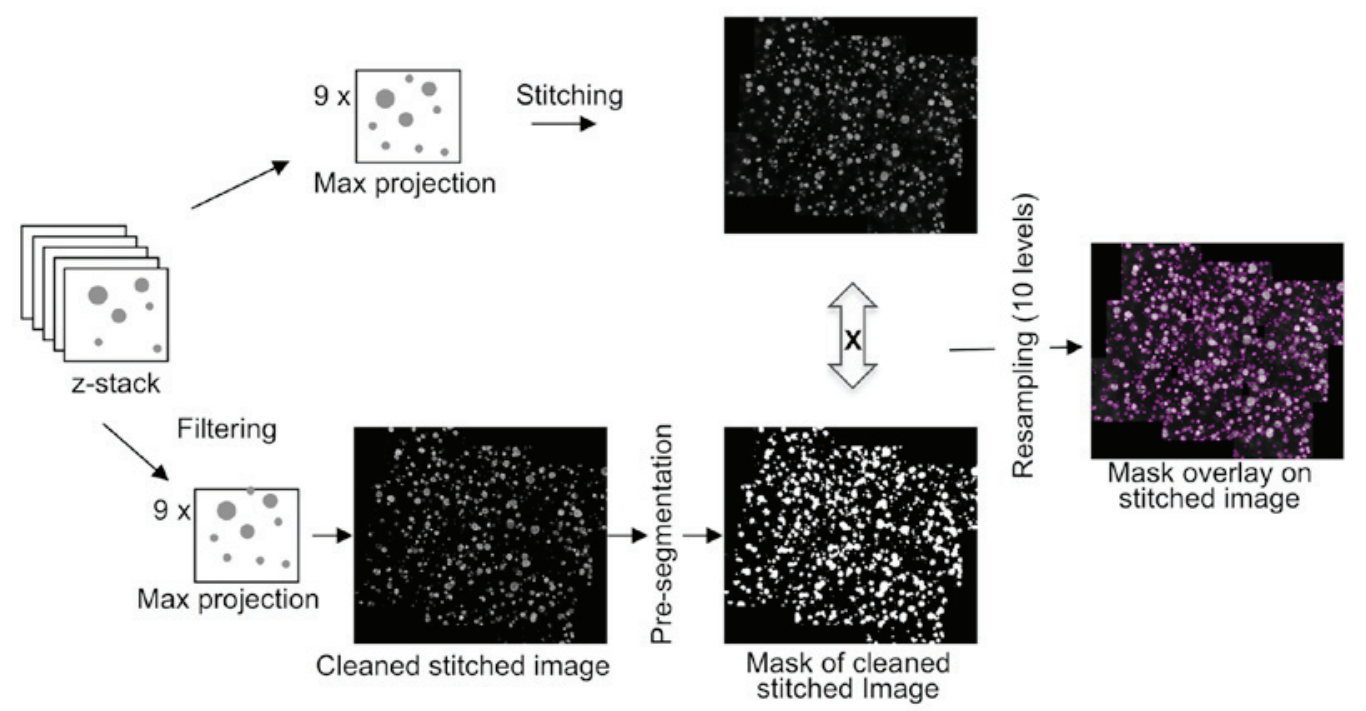

Figure 3. Image processing and feature extraction. A schematic representation of the image processing and feature extraction pipeline. 
A

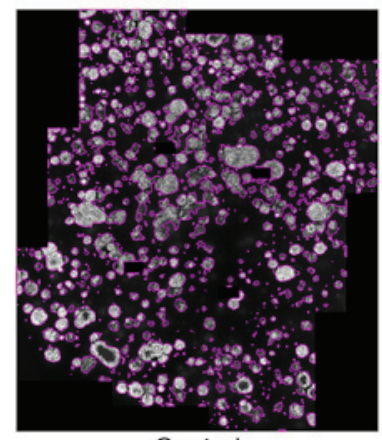

Control

B

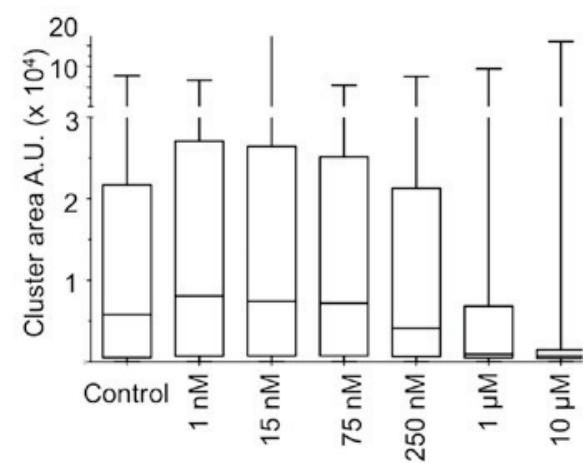

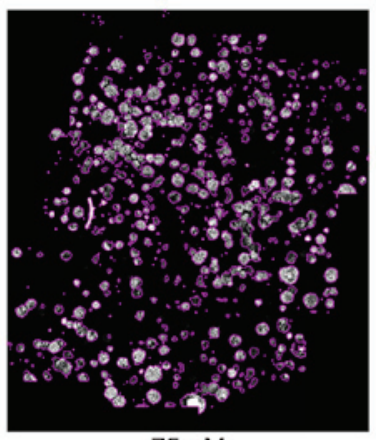

$75 \mathrm{nM}$

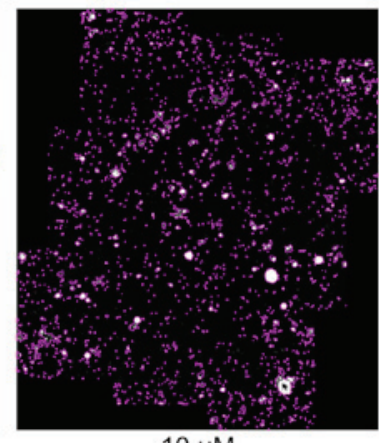

$10 \mu \mathrm{M}$

$\mathrm{C}$

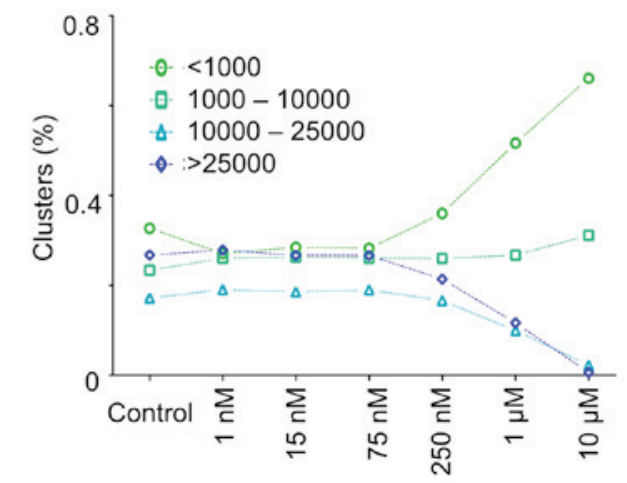

Figure 4. Procedure application to the study the effect of topotecan on HCT116 colorectal carcinoma cells grown in a 3D Biomimesys ${ }^{\circledR}$ matrix. Cells were seeded in 3D matrices and imaging was performed at day 9 (i.e. following 3 days of treatment). (A) Global view of microwells following the stitching together of the 9 image tiles for the indicated concentrations of topotecan. (B) Size distribution of cell clusters at increasing concentrations of topotecan. For each concentration, box-and-whisker plots present the median, the quartile and range of each data set. Each plot corresponds to the analysis of the size of clusters from 4-6 wells per concentration. (C) Representation of cluster size by class. The percentage of clusters in each area class (in A.U) was calculated from the total number of clusters from 4-6 wells per concentration. A.U., arbitrary units.

of various sizes were detected and segmented. The middle and right panel show wells treated, respectively, with $75 \mathrm{nM}$ and $10 \mu \mathrm{M}$ topotecan. As evidenced, the segmentation process detects fewer and mostly smaller clusters in a topotecan concentration-dependent manner.

Fig. 4B illustrates the distribution of the size (median, quartile and extreme values) of the detected clusters in these three conditions. The data show that the median decreases in cells treated with $\geq 250 \mathrm{nM}$ topotecan, with a clear decrease of the interquartile range from $1 \mu \mathrm{M}$, thus highlighting a marked decrease of cluster size in response to an increasing dose of topotecan. Cluster size distribution in the control illustrates the great heterogeneity normally observed in such an assay. This heterogeneity is largely lost upon treatment with topotecan and only very small clusters or single cells are detected.

Additional parameters may be extracted from this experiment, including the evolution of the distribution by class of cluster size (Fig. 4C). These data quantitatively reflect the loss of large clusters and the accumulation of aggregates of a very small number of cells, in cells treated with $\geq 250 \mathrm{nM}$.

In conclusion, these results illustrate the power of the approach and capability of this imaging and processing pipeline to detect and accurately quantify the effect of a compound, such as a cytotoxic agent, on a heterogeneous cell population grown in 3D in a hyaluronic acid matrix. Powerful methods for reconstructing and live $3 \mathrm{D}$ in vivo analysis of the process of cancer cell aggregation and growth have already been documented, however, they rely on the use of sophisticated microscopy and/or image processing tools $(14,15)$. The approach reported in the present study aims to provide a simple, robust pipeline to do so based on technologies that are readily available to many investigators.

\section{Acknowledgements}

The present study was financially supported by the CNRS and the University of Toulouse and by a grant (PC201410) from ITMO Cancer AVIESAN (Alliance Nationale pour les Sciences de la Vie et de la Santé, National Alliance for Life Sciences \& Health, Paris) within the framework of the National Cancer Plan. The support of the ITAV imaging facility is gratefully acknowledged and the authors wish to acknowledge the TRI-Genotoul facilities.

\section{References}

1. Ocana A, Pandiella A, Siu LL and Tannock IF: Preclinical development of molecular-targeted agents for cancer. Nat Rev Clin Oncol 8: 200-209, 2010.

2. Lee GY, Kenny PA, Lee EH and Bissell MJ: Three-dimensional culture models of normal and malignant breast epithelial cells. Nat Methods 4: 359-365, 2007.

3. Friedrich J, Ebner R and Kunz-Schughart LA: Experimental anti-tumor therapy in 3-D: Spheroids-old hat or new challenge? Int J Radiat Biol 83: 849-871, 2007. 
4. Hirschhaeuser F, Menne H, Dittfeld C, West J, Mueller-Klieser W and Kunz-Schughart LA: Multicellular tumor spheroids: An underestimated tool is catching up again. J Biotechnol 148: 3-15, 2010.

5. Thoma CR, Zimmermann M, Agarkova I, Kelm JM and Krek W: 3D cell culture systems modeling tumor growth determinants in cancer target discovery. Adv Drug Deliv Rev 69-70: 29-41, 2014.

6. DeVolder R and Kong HJ: Hydrogels for in vivo-like three-dimensional cellular studies. Wiley Interdiscip Rev Syst Biol Med 4: 351-365, 2012.

7. Magin CM, Alge DL and Anseth KS: Bio-inspired 3D microenvironments: A new dimension in tissue engineering. Biomed Mater 11: 022001, 2016.

8. Astashkina A and Grainger DW: Critical analysis of 3-D organoid in vitro cell culture models for high-throughput drug candidate toxicity assessments. Adv Drug Deliv Rev 69-70: $1-18,2014$.

9. Das RK, Pal M, Barui A, Chakraborty C, Ray AK and Chatterjee J: ApoTome to visualize E-cadherin and p63 expression in oral pre-cancer. Biotechnol J 7: 602-607, 2012.
10. Gustafsson MG: Surpassing the lateral resolution limit by a factor of two using structured illumination microscopy. J Microsc 198: 82-87, 2000.

11. Otsu N: A threshold selection method from gray-level histograms. IEEE Trans Sys Man Cyber 9: 62-66, 1979.

12. David L, Dulong V, Le Cerf D, Cazin L, Lamacz M and Vannier JP: Hyaluronan hydrogel: An appropriate three-dimensional model for evaluation of anticancer drug sensitivity. Acta Biomater 4: 256-263, 2008.

13. Preibisch S, Saalfeld S and Tomancak P: Globally optimal stitching of tiled 3D microscopic image acquisitions. Bioinformatics 25: 1463-1465, 2009.

14. Scherer A, Kuhl S, Wessels D, Lusche DF, Hanson B, Ambrose J, Voss E, Fletcher E, Goldman C and Soll DR: A computer-assisted 3D model for analyzing the aggregation of tumorigenic cells reveals specialized behaviors and unique cell types that facilitate aggregate coalescence. PLoS One 10: e0118628, 2015.

15. Lorenzo C, Frongia C, Jorand R, Fehrenbach J, Weiss P, Maandhui A, Gay G, Ducommun B and Lobjois V: Live cell division dynamics monitoring in 3D large spheroid tumor models using light sheet microscopy. Cell Div 6: 22, 2011. 